\title{
Metallographische Mitteilungen aus dem Institut für anorganische Chemie der Universität Göttingen.
}

\author{
XXX. \\ Über die Verbindungen des Mangans mit Silicium. \\ Von
}

Fr. Doerinckel.

Mit 1 Figur im Text and 1 Tafel.

Trotz der technischen Schwierigkeiten, die eine Untersuchung von Mangan-Siliciumlegierungen bietet, widmete ihnen schon ziemlich früh eine grölsere Anzahl von Forschern ihre Aufmerksamkeit. BrunNer ${ }^{1}$ hatte bei der Reduktion eines Gemisches von Manganchlorid und Calciumfluorid mittels Natrium, die er im hessischen Tiegel vornahm, ein Mangan erhalten, das sich durch seine Luftbeständigkeit von dem Devilies unterschied, und WöHLER ${ }^{2}$ hatte erkannt, dals diese Verschiedenheit durch einen Siliciumgehalt, der bis zu $6 \%$ betrug, hervorgerufen wurde. WöHLER suchte nun das Silicium im Mangan anzureichern, indem er Gemische von Manganchlorid mit Fluoriden der Alkalien bei Gegenwart von Salzen der Kieselfluorwasserstoffsäure mittels Natrium reduzierte und erhielt hierbei ein Produkt, dessen Siliciumgehalt bis zu $13 \%$ betrug.

$\mathrm{W}_{\text {ARREN }}{ }^{3}$ erhielt Mangan-Siliciumlegierungen durch Erhitzen eines beliebigen Manganoxyds mit überschüssigem graphitartigen Silicium.

Schon früher hatte SEFsTröM ${ }^{4}$ darauf hingewiesen, dals man durch Reduktion eines Gemisches von Manganoxyden und Kieselsäure mittels Kohle eine $8-10 \%$ Silicium enthaltende Legierung erhält.

1 Pogg. Ann. 101 (1857), 264.

2 Ann. Chem. u. Pharm. 106 (1858), 54.

${ }^{3}$ Chem. News 78, 318.

${ }^{4}$ J. B. Beraelius 10, 123.

Z. anorg. Chem. Bd. 50 . 
VIGouroux ${ }^{1}$ nahm auf Grund von Rückstandsanalysen die Existenz der Verbindung $\mathrm{SiMn}_{2}$ an, die sich beim Zusammenschmelzen der Elemente oder des Siliciums mit Manganoxyd und einem Reduktionsmittel im elektrischen Lichtbogen bilden soll. Er extrahierte die Reguli nacheinander mit kochendem Wasser, verdünnter Salzsäure und Flufssäure und erhielt einen der oben angegebenen Formel entsprechenden Rückstand. Es gelang ihm auch, die genannte Verbindung durch Reduktion eines Gemisches von Kieselsäure und Manganoxyd mittels Aluminiumpulver ${ }^{2}$ darzustellen und ihre Kristalle durch Behandeln des so erhaltenen Produktes mit verdünnter Salzsäure und Flufssäure zu isolieren.

Bei der Reduktion von Kieselsäure, Manganoxyduloxyd und Kalk mittels Kohle im elektrischen Lichtbogen erhielt DE Chacmot ${ }^{3}$ ein Produkt, dem er die Formel $\mathrm{Si}_{2} \mathrm{Mn}$ zuschreibt, das er aber nicht rein darstellen konnte. 'Troost und HaUteredille ${ }^{4}$ schlossen auf die Existenz von stabilen, den Mangankarbiden äbnlichen Mangansiliziden, weil die Komponenten sich unter Wärmeentwickelung vereinigen; die Lösungswärme der Legierungen ist kleiner als die ihrer Komponenten.

In den Rückständen des Roheisens fanden CaRnot und Goutal ${ }^{5}$ nach Einwirkung verdünnter Schwefelsäure ein Mangansilizid der Formel $\mathrm{MnSi}$; wenigstens hatte eine gröfsere Anzahl von Analysen annähernd diese Formel ergeben, doch war es auch ihnen nicht gelungen, das genannte silizid rein zu erhalten.

Endlich nahm P. LEBEAD ${ }^{6}$ eine Reihe von Untersuchungen über Mangan-Siliciumlegierungen vor, um auf Grund von Rückstandsanalysen die Frage, ob bzw. welche Verbindungen beide Elemente miteinander eingehen, zu beantworten. Die Reguli, deren Rückstände er untersuchte, stellte er auf verschiedene Weise dar:

1. Durch Zusammenschmelzen eines Gemisches von Kupfer, Silicium und Mangan.

2. Durch Reduktion von Kalium-Siliciumfluorid und Manganoxyduloxyd mittels Natrium bei Gegenwart von Kupfer.

1 Ann. chim. phys. [7] 12 (1896), 153.

\& Compt. rend. 141 (1905), 722.

${ }^{3}$ Am. Chem. Journ. 18 (1898), 536.

${ }^{4}$ Ann. chim. phys. [5] 9 (1876), 63. 69.

${ }^{5}$ Ann. des Mines [9] 18 (1900), 271.

(Compt. rend. 136 I (1903), 89, 231; Bull. Soc. Chim. [3] 29 (1903), 185; Ann. Chim. Phys. [8] 1 (1904), 553. 
3. Liefs er Kupfer-Siliciumlegierungen auf metallisches Mangan, bzw. auf Manganoxyd bei Gegenwart eines Reduktionsmittels einwirken und

4. liefs er Kupfer-Manganlegierungen auf Silicium einwirken.

Die Reguli wurden gepulvert und abwechselnd mit verdünnter Salpetersäure und Sodalösung behandelt, bis er einen unter dem Mikroskop homogen erscheinenden Rückstand erhielt, den er analysierte. Auf Grund seiner Untersuchungen gibt er die Existenz dreier Mangan-Siliciumverbindungen an: $\mathrm{Mn}_{2} \mathrm{Si}, \mathrm{MnSi}, \mathrm{MnSi}_{2}$.

Die Existenz von Mangan-Siliciumverbindungen war bis jetzt lediglich auf Grund von Rückstandsanalysen angenommen worden; diese allein jedoch sind nicht imstande, eine völlige Aufklärung dieser Frage zu geben, deshalb unternahm ich auf Grund thermischer Analyse die Ausarbeitung eines Zustandsdiagrammes der ManganSiliciumlegierungen.

Der Ausführung der Schmelzversuche stellten sich mannigfache Schwierigkeiten in den Weg. Zunächst greifen Mangan und Silicium im geschmolzenen Zustand Porzellan stark an, so dals stets das Porzellanschutzrohr des Thermoelements mit Platinfolie und Magnesia ${ }^{1}$ geschützt werden mufste. Durch diesen Schutz des Thermoelementes wird der Wärmeaustausch stark erschwert und die Knickund Haltepunkte werden undeutlicher. Eine weitere Schwierigkeit bot die Frage, wie die Schmelzen gegen Oxydation zu schützen seien. Kohlensäure wurde nicht benutzt, um die Bildung von Karbiden bzw. Oxyden auszuschlielsen. Es blieb die Wahl zwischen Wasserstoff und Stickstoff. Von der Verwendung des Wasserstoffes wurde Abstand genommen, weil er bei hohen Temperaturen leicht die Schutzwand des Thermoelementes durchdringt und die Angaben desselben beeinflufst. Es blieb nur der Stickstoff übrig, der zwar bei hohen Temperaturen mit Mangan und Silicium Verbindungen bilden soll; doch konnte ich trotz wiederholter Versuche in keinem Regulus Stickstoff nachweisen.

Von $0-45 \%$ Siliciumgehalt wurde mit konstantem Gewicht: $20 \mathrm{~g}$, von $50-100 \%$ Silicium mit konstantem Volum von $5 \mathrm{ccm}$ gearbeitet. Letztere Malsregel erwies sich wegen des geringen spezifischen Gewicht des Siliciums als notwendig. Der Gewichtsverlust der Reguli beim Schmelzen betrug bei einer Gesamtmenge von $20-13.3 \mathrm{~g}$ der Mischungen im Durchschnitt $0.12-0.17 \mathrm{~g}$

${ }^{1}$ Vergl. Levis und Tammans, Über Mangan-Eisenlegierungen, Z. anorg. Chem. 47 (1905), 136. 
und überstieg nie $0.45 \mathrm{~g}$. Die letzteren grölseren Verluste sind darauf zurückzuführen, dafs die Legierungen sehr fest an den Wänden des Porzellanrohres haften und sich nur schwer völlig davon ablösen lassen. Zur Kontrolle, ob beim Schmelzen eine Konzentrationsänderung eingetreten war, wurden die Reguli von $20 \%, 35 \%$ und $70 \%$ Siliciumgehalt analysiert, die beiden ersteren zeigten einen Manganverlust von $0.15 \%$ bzw. $0.17 \%$, der letztere einen Siliciumverlust von $0.28 \%$. $\mathrm{Da}$ die gefundenen Abweichungen innerhalb der Fehlergrenzen liegen, wurde eine Korrektur der Konzentrationen weder in der folgenden Tabelle noch im Diagramm vorgenommen, die Angaben beziehen sich auf die eingewogenen Mengen. Als Material wurde nach GouDsCHмпDт schem Verfahren dargestelltes Mangan und käufliches Silicium, zu einer grolsen Anzahl von Kontrollversuchen auch ,chemisch reines Silicium" von E. DE HAËN verwandt; letztere Schmelzen sind in der Tabelle und im Diagramm auf der Konzentrationsachse durch ein Kreuzchen bezeichnet.

Die Zusammensetzung der Ausgangsmaterialien ist durch folgende Analysen gegeben:

Mangan nach GoLDschmidt: ${ }^{1}$

$$
\begin{aligned}
& \mathrm{Mn}=99.4 \% \\
& \mathrm{Fe}=0.13 \% \\
& \mathrm{Cu}=0.01 \% \\
& \mathrm{SiO}_{2}=0.43 \%
\end{aligned}
$$

Käufliches Silicium: „chemisch reines" Silicium von E. DE Hä̈s: ${ }^{2}$

$$
\begin{array}{rlrl}
\mathrm{Si} & =98.07 \% & \mathrm{Si} & =98.86 \% \\
\mathrm{Fe} & =0.95 \% & \mathrm{Fe} & =0.42 \% \\
\mathrm{Al} & =0.39 \% & \mathrm{Al} & =0.10 \% \\
\text { Rückstand } & =1.27 \% & \text { Rückstand } & =0.57 \%
\end{array}
$$

Für jede Konzentration wurden zwei Abkühlungs- und eine Erhitzungskurve aufgenommen. Die Temperatur der Knick- und Haltepunkte und die Zeitdauer der eutektischen Kristallisation wurden in der gleichen Weise aus den Erhitzungs- und Abkühlungskurven berechnet wie W. Guertler und G. TaMmanN ${ }^{3}$ es in der Arbeit

1 Vergl. die oben zitierte Arbeit von Levin und 'Tammans über ManganEisenlegierungen.

Vergl. Guertler und Tamann, Die Silicide des Nickels, Z. anorg. Chem. 49 (1906), 93.

${ }^{3}$ Z. anorg. Chem. $45(1905), 207$. 


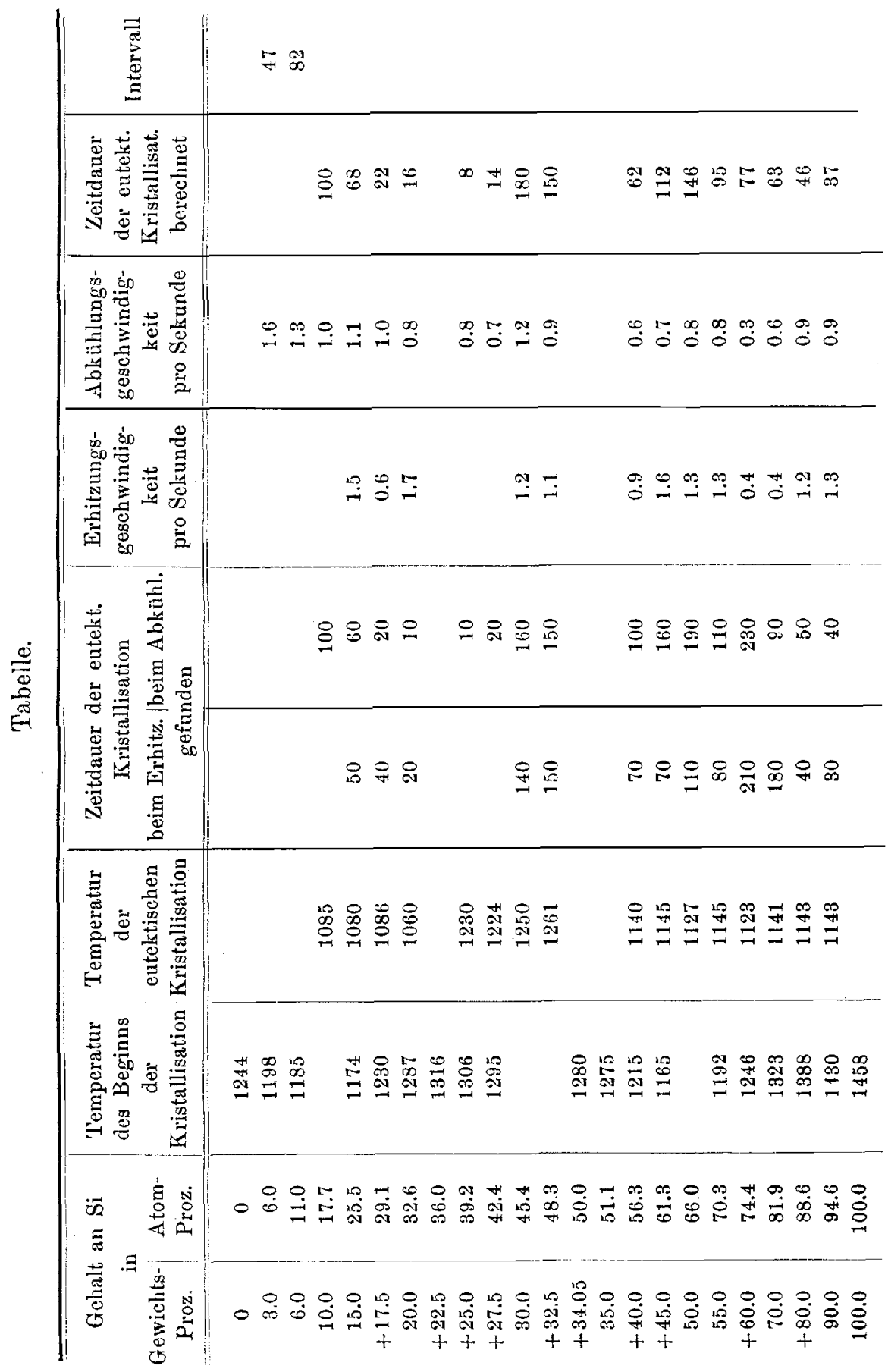


„über die Legierungen des Nickels und Kobalts mit Eisen" beschrieben haben.

Die Resultate der thermischen Analyse sind in der Tabelle auf S. 121 und dem folgenden Diagramm wiedergegeben. Die Temperaturen sind mit Hilfe des Gold- und Nickelschmelzpunktes auf die Skala des Lufthermometers bezogen.

(Siehe Figur, S. 123.)

Der Schmelzpunkt des Mangans wurde in guter Übereinstimmung mit Heraeus ${ }^{1}$ und Levin und Tammann ${ }^{2}$ zu $1244^{\circ}$ bestimmt. Auf Zusatz von wenig Silicium wird der Schmelzpunkt des Mangans erniedrigt, aus der Schmelze scheidet sich aber nicht reines Mangan, sondern eine Reihe von Si-haltigen Mischkristallen aus. Der gesättigte Mischkristall dieser Reihe enthält $10 \%$ Silicium. Die Existenz dieser Mischkristalle wird durch folgende Tatsachen bewiesen: Erstens findet man auf den Abkühlungskurven von $3 \%$ und $6 \%$ Siliciumgehalt deutliche Kristallisationsintervalle, und zweitens bestätigt auch die Struktur der betreffenden Schliffe diese Annahme.

Das nach dem GoLdschMiDTs schen Verfahren dargestellte Mangan besitzt trotz der Geringfügigkeit der Beimengungen merkwürdigerweise keine homogene Struktur. Auf Fig. 1 sieht man die mit verdünnter Flufssäure geätzte Schlifffläche des Mangans. Die hier sichtbare Struktur ist wohl folgendermafsen zu deuten: Zu Beginn der Kristallisation schied sich fast reines Mangan aus, während die geringen Beimengungen von Eisen und Silicium sich in der Schmelze anreicherten und mit derselben zusammen in Form von Mischkristallen kristallisierten. Den an Silicium und Eisen reicheren Partien entsprechen auf Fig. 1 die helleren Teile, während die dunkeln Flecke das reine Mangan bezeichnen.

Die beiden Reguli mit $3 \%$ und $6 \%$ Siliciumgehalt zeigen eine ähnliche Struktur wie das Mangan, der Regulus mit 10\% Silicium besteht aus gröfseren unter sich ziemlich homogenen Kristallen, die mit Ätznäpfchen bedeckt und von zahlreichen Rissen durchsetzt sind. Verbindet man die Temperaturen des Beginnes der Kristallisation für die Schmelzen von $10-30 \%$ Silicium durch eine Kurve, so erhält man die Kurve $B C D$, deren Maximum zwischen $20 \%$ und $22.5 \%$ Silicium, also ungefähr bei $21.3 \%$ liegt. Der Verbin-

1 Zeitschr. f. Elekirochem. 8 (1902).

${ }^{2}$ Vergl, die oben zitierte Arbeit über Mangan-Eisenlegierungen. 
dung $\mathrm{Mn}_{2} \mathrm{Si}$ würde ein Siliciumgehalt von $20.52 \%$ entsprechen. Die Zeitdauer der eutektischen Kristallisation bei $1075^{\circ}$ wird bei $21.0 \%$

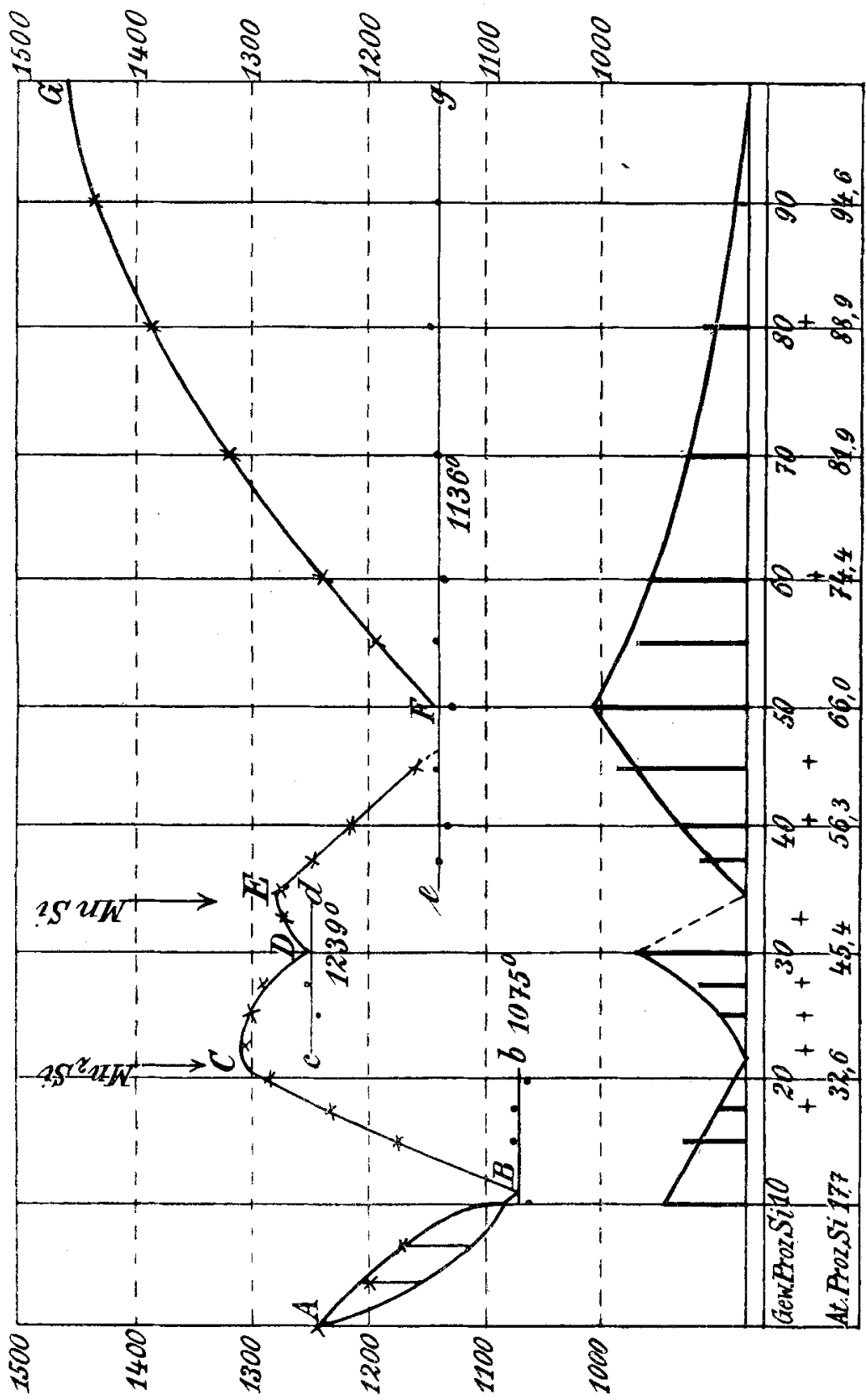


null, die der eutektischen Kristallisation bei $1239^{\circ}$ bei $21.5^{\%}$ Silicium. Das Mittel aus diesen Werten beträgt $21.3 \%$ und weicht also von der Zusammensetzung, die der Formel $\mathrm{Mn}_{2} \mathrm{Si}$ entspricht, nicht erheblich ab.

Entsprechend der Tatsache, dafs die Schmelzen von $0-10 \%$ Silicium eine Reihe von Mischkristallen geben, war zu erwarten, dafs die Kristalle von $\mathrm{Mn}_{2} \mathrm{Si}$ auf dem Kurvenaste $B C$ ron dem gesättigten Mischkristall jener Reihe umgeben wären; es mülsten also in den Regulis von $10-20 \%$ Silicium die primär ausgeschiedenen Kristalle von $\mathrm{Mn}_{2} \mathrm{Si}$ von einem selzundären, in sich röllig homogenen Element umhüllt sein. Nun ergibt aber die mikroskopische Untersuchung, dafs die Struktur jenes sekundär gebildeten Elements eutektisch ist. Fig. 2 zeigt die mit verdünnter Flufssäure geätzte Schliffläche des Regulus von $15 \%$ Silicium. Man sieht, dafs die Menge des primären Elementes die des Eutektikums ungeführ um das fünffache übertrifft. Nimmt man die Konzentration des gesättigten Mischkristalles zu $10 \%$ an, so haben wir die Strecke zwischen $10 \%$ und $21 \%$ in der Weise zu teilen, dafs die Teile den Mengen des Primären und Sekundären proportional sind. Der eutektische Punkt $B$ würde dann auf die Konzentration von etwa $12 \%$ Silicium fallen. Mit steigendem Siliciumgehalt nimmt die Menge des Eutektikums deutlich ab, und in dem Schliff mit $20 \% \mathrm{Si}$ sieht man die letzten Reste desselben zwischen den grofsen Kristallen der Verbindung $\mathrm{Mn}_{2} \mathrm{Si}$. Diese Kristalle zeigen auf den Regulis von $20-30 \%$ Silicium aufserordentlich charakteristische Risse, an denen sie leicht $z u$ erkennen sind. Durch Bildung dieser Risse ist die Behandlung dieser Sehliffe sehr schwierig (Fig. 3).

Zwischen 30-50\% Silicium findet sich ein zweites Maximum auf der Kurve $D E F$, die den Beginn der Kristallisation beschreibt. Es liegt zwischen $32.5 \%$ and $35 \%$ also bei $33.75 \%$, während der Verbindung $\mathrm{MnSi}$ ein Siliciumgehalt von $34.05 \%$ entspricht, die Zeitdauer der eutektischen Kristallisation bei $1136^{\circ}$ wird bei $35 \% \mathrm{Si}$ licium null, es dürfte also hiernach die Existenz der Verbindung MnSi bewiesen sein.

Die Zeitdauer der Kristallisation auf der Horizontalen $D d$ wurde nicht weiter verfolgt, weil diese Eutektische sich nur über ein sehr kleines Konzentrationsintervall erstreckt und ihre Temperatur sich von der des Beginns der Kristallisation nur wenig unterscheidet, wodurch es schwierig wird, die Wärmeeffekte der primären und sekundären Kristallisation auseinander zu halten. Da 
aber die mikroskopische Untersuchung des Regulus mit $32.5 \%$ Silicium noch die rissigen $\mathrm{Kristalle}$ von $\mathrm{Mn}_{2} \mathrm{Si}$, wenn auch in geringer Menge, im Sekundären erkennen liefs, wurde die eutektische Horizontale $D d$ im Diagramm gezeichnet, obwohl ein Haltepunkt, welcher derselben entspricht, nicht mit Sicherheit festgestellt werden konnte.

Die abgerundeten Kristalle der Verbindung $\mathrm{MnSi}$ sieht man auf der Schlifffläche des Regulus von $35 \%$ Siliciumgehalt (vgl. Fig. 4) wo sie fast die ganze Masse desselben bilden; da die Verbindung sehr spröde ist, sind die Kristalle derselben zum Teil ausgesprungen. Die Menge dieser Kristalle ist bei $45 \% \mathrm{Si}$ schon sehr gering.

Mit abnehmender Menge der Kristalle von MnSi wächst die Masse eines anderen Strukturelementes, das sich durch eine schöne parallele Riffelung auszeichnet, und nimmt bei $45 \%$ Siliciumgehalt fast die ganze Fläche des Schliffs ein. Diese Parallelstreifung tritt bei schwachen Ätzen mit verdünnter Flufssäure sehr deutlich und scharf hervor. Die ein wenig verwischten vertikalen Parallelstreifen (Fig. 5) entsprechen dieser Riffelung. Diese Kristallart ist neben den primären Siliciumkristallen in allen Schliffen von 50-100\% Silicium vorhanden. Der Regulus mit 50\% Silicium enthält, wie man auf Fig. 6 sieht, schon eine erhebliche Menge von primär ausgeschiedenem Silicium neben der durch ihre Parallelstreifung ausgezeichneten Kristallart. Die Streifung ist nur bei starker Vergröfserung zu sehen und tritt auf Fig. 6, die mit nur 80 facher Vergrölserung aufgenommen ist, nicht hervor. Die Zusammensetzung dieser charakteristisch gezeichneten Kristalle kann auf Grund thermischer Analyse nicht angegeben werden. Es ist sehr wahrscheinlich, dals die Schmelzkurve zwischen $45-50 \%$ Silicium ein freies oder verdecktes Maximum aufweist, das aber in einem Konzentrationsintervall von $5 \%$ bei sehr geringen Temperaturunterschieden nicht ausgearbeitet werden konnte. Es ist sehr wahrscheinlich, dafs das geriffelte Strukturelement sich direkt aus der Schmelze bildet, denn nach dem Erhitzen des Regulus mit $45^{\circ} \%$ Silicium auf $1120^{\circ}$ und Abschrecken desselben in Wasser konnte auf der Schlifffäche des noch spröder gewordenen Regulus die geriffelte Kristallart deutlich in unveränderter Menge wiedererkannt werden. Dafs die Zusammensetzung dieser Kristallart der Formel $\mathrm{MnSi}_{2}$ entspricht, ist wohl ausgeschlossen, da der Regulus mit $50 \%$ Silicium bereits deutlich primär ausgeschiedene Siliciumnadeln zeigt, und die angeführte Formel einen Siliciumgehalt von $50.81 \%$ erfordern würde. 
Die Kristallisation der Schmelzen von 50-100\% Silicium ist einfach: Auf der Kurve $F G$ scheidet sich primär Silicium aus, während bei $1136^{\circ}$ das gestreifte Strukturelement von unbestimmter Zusammensetzung kristallisiert.

Das Mangan bildet also mit Silicium zwei wohl definierte Verbindungen: $\mathrm{Mn}_{2} \mathrm{Si}$ und $\mathrm{MnSi}$, es existiert ferner eine Reihe von Mischkristallen von reinem Mangan an bis zu einem Gehalt von 17.7 Atomprozenten Silicium, und schliefslich ergab die mikroskopische Untersuchung zwischen 37.5 und 100 Gewichtsprozenten Silicium das Vorhandensein eines durch schöne Parallelstreifung ausgezeichneten Strukturelements, dessen Zusammensetzung nicht bestimmt werden konnte.

Eine Analogie im Verbalten von Eisen und Mangan dem Silicium gegenüber ist nicht $\mathrm{zu}$ verkennen. Während GUERTher und TAMManN bei Eisen-Silicium eine Reihe von Mischkristallen von reinem Eisen bis zur Zusammensetzung $\mathrm{Fe}_{2} \mathrm{Si}$ konstatierten, fand ich bei Mangan-Silicium eine analoge Reihe von Mischkristallen von Mangan bis zu 17.7 Atomprozenten Silicium. Den Verbindungen $\mathrm{Fe}_{2} \mathrm{Si}$ und FeSi stehen die Verbindungen $\mathrm{Mn}_{2} \mathrm{Si}$ und $\mathrm{MnSi}$ gegenüber.

Herrn Prof. Dr. G. Tammann möchte ich zum Schlufs für die Anregung zu dieser Arbeit und seine vielseitige Unterstützung bei derselben meinen verbindlichsten Dank aussprechen.

Göttingen, Institut für anorganische Chemie der Universität.

Bei der Redaktion eingegangen am 7. Juni 1906. 